\title{
A context-aware framework for the efficient integration of femtocells in IP and cellular infrastructures
}

\author{
Prodromos Makris ${ }^{1 *}$, Nikolaos Nomikos ${ }^{1}$, Dimitrios N Skoutas ${ }^{1}$, Demosthenes Vouyioukas ${ }^{1}$, Charalabos Skianis ${ }^{1}$,
} Jie Zhang ${ }^{2}$ and Christos Verikoukis ${ }^{3}$

\begin{abstract}
In today's heterogeneous networking (HetNet) environments, where end users are provided with universal connectivity opportunities, femtocell deployments can become key players in the enhancement of critical performance indicators such as capacity, coverage, QoS, etc. In order to confront the up-to-date LTE femtocell challenges, we propose a context-aware framework that provides a controlled environment from the femtocell point of view, which is required for applicable functionality. More specifically, we aim to (a) control the local environment where the femtocell is placed within, by efficiently managing the total incoming traffic load and by continuously adjusting the distribution of the backhaul capacity among the coexisting networks and (b) control the macro-femto interference caused by macrocell users transmitting close to the femtocell by investigating the "femtocell as a relay" concept. Finally, the performance of the proposed framework is evaluated via simulation results showing that the overall performance of a HetNet environment can be leveraged in terms of QoS requirements, energy saving and data rate enhancement.
\end{abstract}

Keywords: Context-aware, Femtocell, Relay, RRM, HetNet

\section{Introduction}

The Future Internet (FI) vision is expected to extend the "Always Best Connected" (ABC) notion and include use cases according to which the FI end user will attain any service on a single intelligent device (e.g., smartphone, tablet, etc.) using any available network within a heterogeneous network environment consisting of open, cognitive, and collaborative wireless and wireline networks. Towards this direction, heterogeneous networking (HetNet) deployments, proposed by 3GPP LTE-A standardization group, are expected to become a reality in the next few years, thus providing efficient ways to deal with the continuously growing traffic demand $[1,2]$. In contrast with the widely known cellular networks, which are based on a dedicated terrestrial backbone, the emerging femtocell technology utilizes the IP backhaul network along with small-sized base stations (BSs) [3].

\footnotetext{
* Correspondence: pmakris@aegean.gr

'Department of Information and Communication Systems Engineering, University of the Aegean, Karlovassi Samos GR-83200, Greece Full list of author information is available at the end of the article
}

Hence, the advent of femtocells added a new perspective on the $A B C$ concept as they are capable of extending cellular coverage with minimum cost by utilizing an already deployed IP infrastructure. However, despite their mature cellular heritage, several aspects of such technology still require detailed investigation [4]. Indeed, merging the strict QoS requirements of femtocell users with the best effort IP architecture can be problematic. The main issue is the efficient sharing of the common backhaul capacity among the femtocell and other existing wireline or wireless networks [5]. As IP networks do not employ a Service Admission Control (SAC) operation, the capacity allocated to the femtocell can significantly and unpredictably fluctuate over time. Moreover, the backhaul capacity itself can also fluctuate as a result of traffic load variations at the ISP core network. Consequently, the performance of the femtocell operation can considerably be degraded, thus leading to inability to maintain the required QoS [6]. Current femtocell deployments address this issue by operating in closed mode, thus limiting the number of users that can

\section{实}


have access to the femtocell. This approach represents a low-cost and low complexity solution, which, however, can be efficient only under low traffic conditions. On the other hand, future LTE femtocells operating in a heterogeneous and small cell network (HetSNet) environment may widely function in open or hybrid access modes and therefore need to handle significantly higher traffic loads [7].

Furthermore, the unstructured placement of femtocells within a structured cellular network causes interference issues. In the downlink, the femto base station (BS) transmitting towards its femto users will interfere with the reception of nearby macro users served by the macro BS. On the other hand, in the uplink, users transmitting towards the macro BS may cause interference to closely located femtocells [8]. As the positioning of the femtocells cannot be controlled, the only source of interference that can actually be controlled is the transmission energy of the mobile terminals (MTs) that communicate with the macrocell BS (e.g., following femtocell as a relay concepts described in this article). Consequently, we focus on the uplink case aiming to reduce the transmission power of the macro user equipments (UEs) in order to protect the operation of nearby femtocells. Towards this end, one state-of -the-art proposal is that each femtocell should serve its own interfering macro UEs [1]. Although this practice would definitely reduce interference, on the other hand it may raise privacy and QoS issues, as the femtocell owners will not always be willing to share their limited backhaul line with external users.

Based on the above considerations, we propose a context-aware framework for LTE femtocells' efficient integration (CA-FEI) in IP and cellular infrastructures, which aims to provide a "shielded" environment for the femtocell operation. Our approach is twofold: (a) control the local environment, where the femtocell is placed within, by efficiently managing the total incoming traffic load and continuously adjusting the distribution of the backhaul capacity among the coexisting networks. The full exploitation of femtocells' backhaul capacity is examined following the context-aware mobile and wireless networking rationale [9] aiming to achieve more accurate and timely radio resource management (RRM) decisions and (b) to confront the uplink interference that comes from macro UEs by exploiting the use of the "femtocell as a relay" concept. We identify when a femtocell is able to act as a relay in order to assist the reduction of local interference and thus improve the QoS in other femtocells of the same region.

The remainder of the article is organized as follows: in Section 2, we cite the related work in the areas that make up the subject of our study, in Section 3, a typical system model is presented together with an outline of the proposed modifications, in Sections 4, 5, 6, and 7, the functions that should be implemented by each CA-FEI module are presented in detail, while in Section 8, numerical results and details about the simulations are provided. Finally, concluding remarks are given in Section 9.

\section{Related work}

Several QoS considerations related to the femtocell network architecture have arisen and deal with the question how it is possible for the backhaul network to provide acceptable QoS [4]. The existence of net heterogeneity poses a serious concern, especially in cases where the wireline backhaul provider is not in a tight strategic relationship with the cellular operator. Moreover, as femtocell access points (FAPs) are part of a continuously changing HetNet environment [1], femtocells experience difficulty transferring even low-bandwidth services.

\subsection{Integration of femtocell and IP traffic}

The limitations of XDSL backhaul capacity is a vital issue that needs to be addressed. Recent 3GPP standardization activities assert that it shall be possible for the network to set different criteria for access control in a hybrid cell for a closed subscriber group (CSG) and non-CSG members [10]. These different criteria incur conflicting results and this problem can be tackled by specific admission control policies, as addressed in our related work in [11]. To the best of the authors' knowledge, there is no other work in the literature dealing with the efficient integration of femtocell and IP traffic. The proposed CA-FEI framework can be considered as complementary to [12], which presents a scheme for dynamic service-level agreement negotiation between the ISP provider and the femtocell operator, aiming to tackle the problem of XDSL line's varying capacity. In general, our proposed scheme adopts a hybrid partitioning policy [13] and can be classified within the family of admission control schemes designed for the integration of heterogeneous networks, such as [14-17]. However, such schemes are not directly applicable in our case as either (a) they consider significantly different system models, which in most cases include networks that have their own separate links to the core network or (b) they support basic QoS features that are not compatible with the strict QoS requirements of a femtocell.

\subsection{Femtocells as relays and interference mitigation}

In the classic setup, a femtocell relays the traffic of the femto users utilizing the wired backhaul link. In more advanced cases, they could adopt an open access policy and cooperate with users served by the macro BS. In [8], the two different policies are compared concluding that in TDMA/OFDMA networks, the decision of whether a macro UE will be accepted in a hybrid access femtocell, depends on user density. Elkourdi and Simeone [18] suggest the use of femtocells as relays in the uplink stream of a cellular network by serving both indoor and outdoor 
users via the wireline backhaul. The decoder is positioned at the mobile operator network and soft information is provided by the femtocell/relay to facilitate decoding. Rath et al. [19] suggest utilizing users, in the range of the femtocell, as relays of information to the macrocell UEs and conclude that with this system architecture, the macrocell's bandwidth shortage can be surpassed. Dizhi and Wei [20] elaborate on the relaying concept of [19], employing femtocells with the ability to broadcast relay service availability to the macrocell users on behalf of the femtocell users. Moreover, it is shown that the proposed architecture achieves better load balancing for the macrocell and improved throughput for the macrocell users. Furthermore, in [21], mitigation schemes using femtocells with relaying capabilities are presented. Finally, the proposed scheme of [22] introduces femtocells that are able to decode the control channel of the macrocell BS. In this way, the femto BSs can adjust their transmissions, since they have information about the scheduled users in the macrocell avoiding inter-cell interference with the macrocell. In addition, interference cancellation is implemented at the femtocells as an extra interference mitigation measure.

To the best of the authors' knowledge, no work studies the concept of merging classical relaying and femtocell technologies aiming to reduce the burden of the wireline backhaul. Consequently, we introduce the concept of femtocells as relays that can communicate with a wireless backhaul with the macro BS, allocating as a result, all the available wireline backhaul to the femto UEs (see also paper's contribution points in Section 1).

\section{System architecture and proposed modifications}

In the following, we will present a typical topology of a femtocell deployment, which will help us to clearly point out the proposed architectural modifications.

\subsection{Typical topology of a femtocell deployment}

As shown in Figure 1, the main network elements of a typical femtocell deployment are the FAP itself and a broadband IP router that allows FAP to access the Internet and consequently the Femtocell GateWay (FGW), which serves several FAPs. The communication between the FAP and the FGW is performed through the Iu-h interface while the Iu-Cs (Circuit switched) and Iu-Ps (Packet switched) interfaces are used for the communication between the FGW and the Mobile Core Network [23]. As it also becomes apparent from the network topology depicted in Figure 1, the router may also serve a number of wired LAN users as well as a number of wireless users through an attached Wi-Fi AP. Furthermore, according to the scenario being assumed, the FAP should be able to serve concurrently from 4 to 32 UEs and provide them with the same services and the same QoS as a typical Node B.

\subsection{Overview of the proposed modifications}

The proposed CA-FEI scheme (Figure 2) is practical, as it does not require any modifications to the existing IP infrastructure. Actually, it can be realised as an add-on unit, placed on top of the existing networks, in the form of an integrated IP Router/FAP. Furthermore, a Decodeand-Forward relay module gives the ability to this integrated unit to act, when possible, as a relay for the macrocell users.

\subsubsection{RRM integration layer}

The RRM part of CA-FEI is composed of two robust, efficient and low complexity modules, which can easily be implemented and directly applied to real-market femtocell deployment scenarios. The first module is an Integrated SAC (ISAC) frontend, which is based on the effectiveness of the well-known capacity partitioning rationale, while it is complemented with a novel periodic

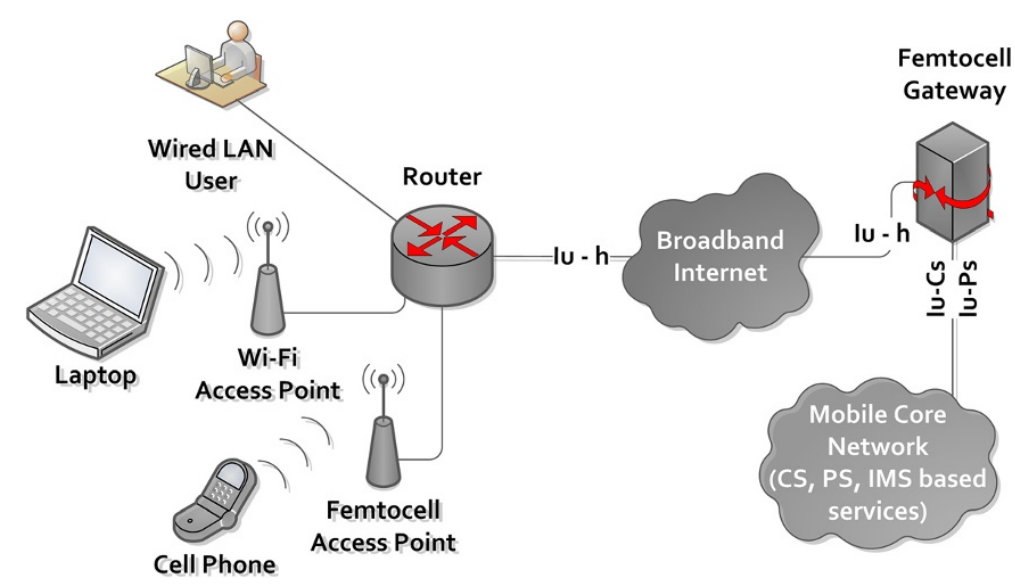

Figure 1 Typical topology of a femtocell deployment. 


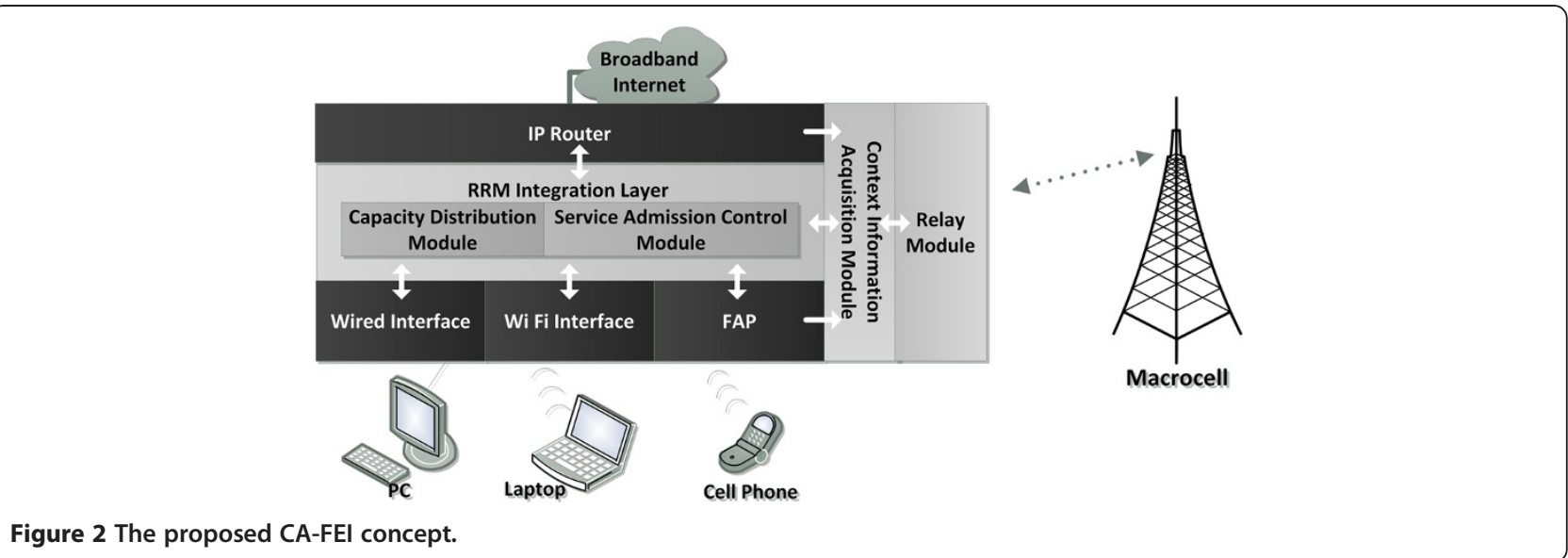

partition adjustment (PPA) process that addresses any backhaul capacity fluctuations as well as variations of traffic load composition. Although our main concern is to preserve the QoS of delay sensitive services originating from the femtocell, we also took into account that similar services may originate from other network interfaces and, therefore, they have to be treated equally. Hence, ISAC was designed to operate over a single virtual network interface, thus applying the same admission policies to the total incoming traffic load. Subsequently, the capacity management (CAM) module interprets the ISAC admission decisions to the actual capacity distribution among the real physical network interfaces (Figure 3).

\subsubsection{Context information acquisition module}

The context information acquisition module (CIAM), as shown in Figure 2, acquires low-level data from the IP router and the network interfaces, and transforms them to the proper form (i.e., high-level context information) in order to be utilized as input for the SAC and Capacity Distribution modules as well as for the Relay module (cf. Section 6). The main functionalities of CIAM regarding context acquisition are the initial set-up, monitoring, and gathering of context. The setup phase regards the setting of the initial operating parameters, while the monitoring and gathering processes monitor the statistical properties of the incoming traffic load and backhaul capacity in order to adjust, if necessary, the parameters originally selected by the user and to trigger the operation of the relay module.

\subsubsection{Decode-and-forward relay module}

An additional functionality implemented in the proposed framework is decode-and-forward relaying. Contrary to the classic setup (see Figure 1), we use wireless backhaul links towards the macro BS by adding an outdoor donor antenna connected through cable link with the femto BS. In this way, we integrate wireless relaying capabilities to the femtocells in order to protect the communication of the femtocell BS and the femto UEs using the wired backhaul link. Furthermore, as the macro UE communication with the femto BS will be performed on small distance links, there is an increased possibility of achieving a reduced power transmission in this part of the macrocell. This fact can lead to better SINR in other femtocells in the vicinity of the macro UE, which were not available to cooperate at that time. Also, as the

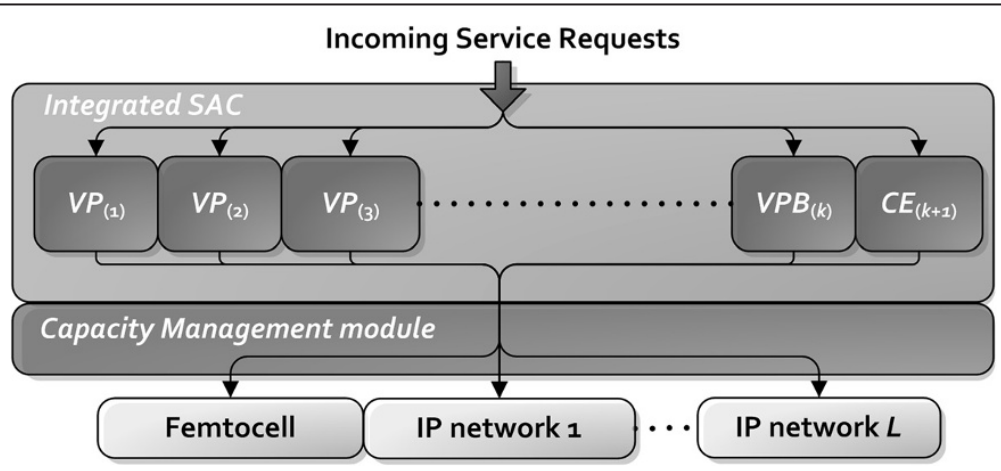

Figure 3 The RRM part of CA-FEI. 
second hop is performed via an outdoor antenna on the rooftop of the building, there is a good chance of having LOS condition with the macro BS. As a result, the required power in the second hop can also be reduced, while attaining the desired data rate in the end-to-end two-hop link.

\section{SAC module}

In order to perform the ISAC operation, the definition of common service classes is required for all the underlying networks. Thus, services with similar characteristics, regardless of their network origin, are classified to the same integrated service group (ISG). The number of ISGs should be at least four, corresponding to conversational, streaming, interactive, and background service classes as determined by 3GPP [23]. However, each ISG can be further partitioned, if a more precise categorization is required. In the general case, corresponding to a total of $k I S G$ s, the backhaul capacity is also divided to $k$ primary virtual partitions $(V P s)$ and each partition $S_{j}, 1 \leq j \leq k$ is associated to the $j$ th $I S G_{j}$. An additional $(k+1)$ partition, namely the Capacity Exchange (CE) partition, is also defined as required by the PPA process (cf. Section 4.2). A prerequisite for CA-FEI is that a method for indicating the QoS requirements of each traffic flow, such as DiffServ [24], should be employed at all coexisting networks. However, if a flow does not need any QoS or does not indicate that it needs a specific QoS treatment, then it will be treated as a background service (cf. Section 4.2.3).

\subsection{Capacity partitioning}

The aim of ISAC is to ensure that a service call with specific QoS requirements is admitted to the system only if there is adequate capacity to handle it. Consequently, the initial virtual capacity partitioning should be performed for the worst-case situation, where the traffic load intensity $A_{j}$ offered by each $I S G_{j}$ has its maximum value, while at the same time the backhaul capacity has its minimum value $C_{\min }$. During this critical system state, the $V P$ of the delay insensitive background services, denoted in the following as $V P B$, is restricted to its minimum size. Assuming that $V P B$ is the $k$ th partition, then the partitioning problem is constrained to the first $k-1 V P$ s.

Let $a_{j}$ be the average rate requested by services of $I S G_{j}$, which we assume that arrive following a Poisson process with exponentially distributed holding times. Then, the initial size $S_{j}^{0}$ of partition $V P_{j}$ can be defined as a multiple of $a_{j}\left(S_{j}^{0}=m_{j} \times a_{j}, m_{j} \in \mathbb{Z}^{+}\right)$and for a given value of $m_{j}$ the blocking probability $P_{b, j}\left(A_{j}, m_{j}\right)$ for the members of the $I S G_{j}$ class can be computed by the Erlang $B$ formula. If $G_{j}$ is the Grade of Service (GoS) required for
$I S G_{j}$ services, then the overall partitioning problem can be described as the definition of the vector $m_{j}=\left(m_{1}, m_{2}\right.$, $\left.\ldots m_{k-1}\right)$, which satisfies inequalities (1) and (2):

$$
\begin{aligned}
& P_{b, j}\left(A_{j}, m_{j}\right) \leq G_{j}, \quad \forall j \in[1,(k-1)] \\
& \sum_{j=1}^{k-1}\left(S_{j}^{0}\right) \leq C_{\min }
\end{aligned}
$$

Although, in the general case, the above set of equations has multiple possible solutions, the initial size of the partitions can be defined through a straightforward process that ensures a reliable operation in the worstcase scenario.

(a) At first, the size of each of the $k-1$ partitions has to be defined in such a way that the required upper bound $G_{j}$ of the blocking probability of the $I S G_{j}$ calls to be guaranteed. Therefore, as the initial size of the partitions should correspond to the maximum acceptable blocking probability, each $S_{j}^{0}$ can be calculated directly from Equation (1) for the case of equality (i.e., $P_{b, j}\left(A_{j}, m_{j}\right)=G_{j}, \forall j \in[1$, $(k-1)])$.

(b) Subsequently, we have to ensure that the summation of the calculated capacities $S_{j}^{0}$ of the partitions meets the limitation of Equation (2). If not, then the network administrator has to select and prioritize those ISGs for which the target blocking probability can be further increased. The administrator has to take into account both the nature of the network and the requirements of the users so that his decision to be as tailored as possible to the specific network environment. In case the femtocell is installed by a home user, then he should be guided by a properly designed setup process in order to make the right selections based on his preferences (cf. Section 6.1). Given the prioritized list of the selected ISGs, we iteratively follow the order of the list, and at each iteration we reduce by $a_{j}$ the size of each partition $S_{j}^{0}$ until Equation (2) is valid.

(c) Finally, if a part $C_{L}$ of the $C_{\min }$ capacity is left unallocated after the initial partitioning process, then it is shared among the $j$ partitions proportionally to their respective traffic load intensity $A_{j}$. Thus, each partition is extended by a fraction of $C_{L}$, which is normalized to $a_{j}$. The initial size $S_{j}^{0}$ of each partition $V P_{j}$ can be defined as

$$
S_{j}^{0}=\left(m_{j} \cdot a_{j}\right)+\left(l_{j} \cdot a_{j}\right)=\left(m_{j}+l_{j}\right) \cdot a_{j}
$$

where $l_{j}$ is an integer, which is calculated by applying the floor function as follows: 


$$
l_{j}=\left(A_{j} / \sum_{i=1}^{k}\left(A_{i}\right)\right) \cdot C_{L} \cdot \frac{1}{a_{j}}
$$

The additional capacity is calculated consecutively starting from the partition that is expected to handle the higher traffic load while ties are randomly resolved.

\subsection{PPA}

While the initial capacity partitioning guarantees a reliable operation for the worst-case scenario, at the same time it could lead to capacity underutilization under normal traffic conditions. Therefore, we employ the PPA process in order to sequentially transfer unutilized capacity from the $k-1$ primary $V P s$ to the $C E$ partition and vice versa. Consequently, the VPs' size is not fixed, but instead it is adjusted in response to the variations of the traffic load composition.

\subsubsection{Size of VPs}

Starting from $V P_{1}$ towards $V P_{k-1}$, the PPA process, at time intervals of $\Delta t$, sequentially adjusts the size of all the $V P_{\mathrm{s}}$. Thus, the size $S_{j}(t)$ of the $V P_{j}$ partition is adjusted by a term $w_{j}(t)$ :

$$
S_{j}(t)=\min \left\{\left[S_{j}(t-\Delta t)+w_{j}(t)\right], S_{j}^{0}\right\}
$$

Therefore, as implied by Equation (5), the upper bound of $S_{j}(t)$ is set to $S_{j}^{0}$ (cf. Equation 3$)$ ). The adjusting function $w_{j}(t)$ is equal to a negative or positive step of $a_{j}$ depending on the blocking rate $B_{j}$ that the $I S G_{j}$ service calls have experienced, averaged over a time interval of $d$ seconds, and the current occupancy $O_{j}$ of the partition, namely:

$$
w_{j}(t)=\left\{\begin{array}{c}
-a_{j} \text { if }\left[B_{j}(t-d)<G_{j}\right] \text { and }\left[O_{j}(t) \leq S_{j}(t)-g_{j} \cdot a_{j}\right] \\
0 \text { if }\left[B_{j}(t-d)<G_{j}\right] \text { and }\left[O_{j}(t)>S_{j}(t)-g_{j} \cdot a_{j}\right] \\
a_{j} \text { if }\left[B_{j}(t-d) \geq G_{j}\right]
\end{array}\right.
$$

The higher the value of $d$ is, the more averaged over time $B_{j}$ becomes. The guarding factor $g_{j}$ is a positive integer $\left(1 \leq g_{j} \leq m_{j}\right)$, which guarantees that the size of the partition can be decreased only if it has at least $g_{j} \cdot a_{j}$ available capacity for the future incoming $I S G_{j}$ service calls.

Furthermore, the size of a $V P$ can be increased by $a_{j}$ only if a corresponding decrease of the $C E$ partition by $-a_{j}$, according to its occupancy, can be performed. If not, the capacity adjustment of the $V P$ is scheduled and performed asynchronously, when conditions permit together with the inverse adjustment of the $C E$.

\subsubsection{Size of the CE partition}

The total capacity adjustment for the $C E$ partition is the negative of the summation of all the $w_{j}(t)$ adjustments calculated for the $V P$ s. Thus, the required capacity $S_{C E}^{R}(t)$ for the $C E$ partition is

$$
S_{\mathrm{CE}}^{R}(t)=S_{\mathrm{CE}}^{R}(t-\Delta t)+\sum_{j=1}^{k-1}\left(-w_{j}(t)\right)
$$

However, the CE partition is not only used as a CE point between the VPs, but also acts as a common pool for all the ISGs. As a result, the actual capacity $S_{\mathrm{CE}}(t)$ of the $C E$ partition cannot become less than the currently occupied capacity $O_{\mathrm{CE}}(t)$

$$
S_{\mathrm{CE}}(t)=\max \left\{S_{\mathrm{CE}}^{R}(t), O_{\mathrm{CE}}(t)\right\}
$$

As previously mentioned, the adjustment steps, which cannot be performed due to the restriction of Equation (8), are scheduled and performed asynchronously.

\subsubsection{Size of the background services virtual partition (VPB)}

Based on the previous steps, the partitioning process up to this point is dimensioned on the minimum backhaul capacity $C_{\min }$. The difference between $C_{\min }$ and the current value of the backhaul capacity $C(t)$ forms the $V P$ $k$ for the background services

$$
S_{\mathrm{VPB}}(t)=C(t)-C_{\min }(t)
$$

Obviously, the background services can be accepted either at the $V P B$ partition or at the $C E$ partition.

\subsection{Admission decision process}

As long as the virtual capacity partitions are defined, the admission decision process, as shown at Figure 4, is straightforward. An incoming service call of the $I S G_{j}$ is accepted only if (a) there is an adequate free capacity at the respective $j$ th virtual partition or else (b) if there is an adequate free capacity at the $C E$ partition. Thus, the ISAC module ensures that (i) all service requests are handled equally irrespective of the employed network interface and that (ii) there is always adequate backhaul capacity to handle all the admitted service calls with their respective QoS requirements.

Consequently, ISAC extends the QoS-aware admission control of femtocells to all the underlying IP networks following the FI paradigm discussed at [25]. Furthermore, ISAC does not require the ISP to exclusively provide any kind of QoS guarantees. On the contrary, the main admission policy of ISAC is to limit the acceptance of QoS demanding services to what the ISP is proven to be able to provide. After a service call is accepted and has been allocated capacity at one of the VPs, it is then forwarded to the CAM module. 


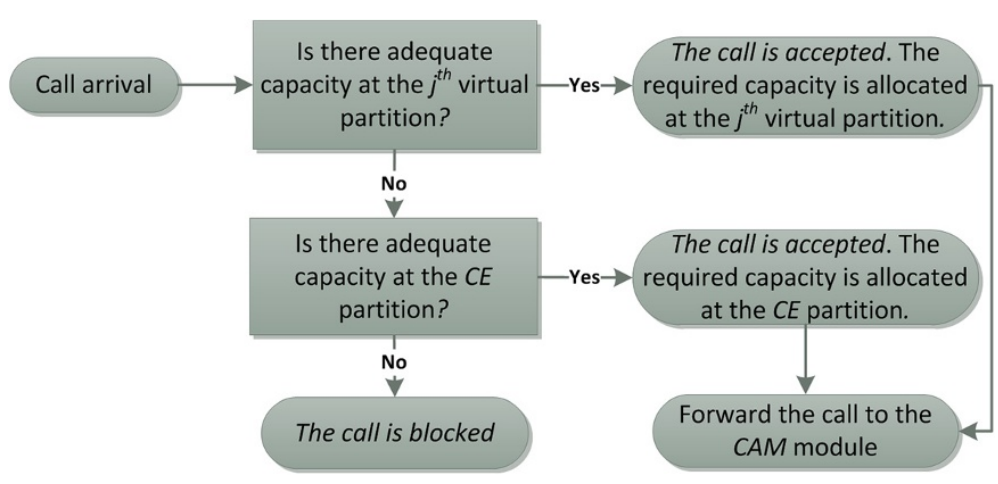

Figure 4 Admission decision process.

\section{CAM module}

The CAM module is responsible to route an admitted service call to the appropriate serving network and to adjust the backhaul capacity distribution among all the underlying networks. Specifically, assuming a total of $L$ underlying networks (see Figure 3) each one having $N_{y}$ $(1 \leq y \leq L)$ ongoing service calls of average requested rate $a_{i}\left(1 \leq i \leq N_{y}\right)$, the capacity $U_{y}(t)$ allocated to each network is defined as follows:

$$
U_{y}(t)=\left(\sum_{i=1}^{N_{y}} a_{i} / \sum_{z=1}^{L} \sum_{i=1}^{N_{z}} a_{i}\right) \cdot C(t)
$$

Hence, each admitted service call modifies the fraction of the current backhaul capacity, which is allocated to each serving network. Consequently, while the ISAC module is based on the minimum value of the backhaul capacity in order to be able to guarantee a minimum QoS for all admitted service calls, the CAM module distributes the total available backhaul capacity among the serving networks.

In other words, although a VP may be empty and reserved for a specific ISG, its respective physical capacity does not remain unused but instead can always be utilized by the underlying packet schedulers, as implied by Equation (10). Therefore, it becomes clear that designing CA-FEI as a two-tier scheme makes it possible to have, at the same time, a virtual (logical) capacity distribution for the admission control process and an actual (physical) capacity distribution for the serving networks. As a result, the proposed CA-FEI scheme is more flexible compared to typical one-tier schemes that utilize actual capacity partitions and thus it is able to achieve higher utilization of the backhaul capacity.

Furthermore, it can also be concluded from Equation (10) that if two networks have to serve the same traffic load, in terms of volume and distribution among the ISGs, these networks will also be allocated the same bandwidth. Consequently, the bandwidth distribution among the serving networks can be considered as fair because it is directly proportional to their ongoing traffic load.

\section{CIAM}

The CIAM described in this section aims to transform low-level data acquired from the IP router and the various network interfaces into higher-level context input information for the RRM modules as well as for the relay module, while the latter can also provide to CIAM context information regarding the radio access links' quality in the vicinity of the femtocell. For example, in cases where CIAM identifies high wired backhaul capacity utilization, it can trigger the relay module to route delay sensitive traffic or traffic incurred by macro UEs via the wireless backhaul link. On the contrary, when the relay module informs CIAM that the wireless backhaul link is congested or the femtocell's radio environment experiences high interference levels, CIAM can inform the RRM integration layer in order the various partitions' capacities to be restructured in ways that efficient QoS can be achieved for all ISGs. CIAM has two main functionalities regarding context acquisition, which are initial set-up phase and monitoring/gathering of context.

\subsection{Initial setup phase}

During the deployment phase, the user (i.e., home user or ICT technician) will be able to set, through a GUI, the initial operating parameters. Such parameters are the number of users that is expected to be served by the femtocell as well as their preferences regarding the services they usually use and the way they use them. As this can be a difficult task for the average home user, he will be offered, through the setup GUI, a set of typical values to select from. More advanced setup procedures may even autonomously select one of these sets through a question-answer process interacting with the user, aiming to discover his preferences. Furthermore, an "Advanced user mode" will allow more experienced users or ICT specialists to finely tune the overall process. The 
result of this initial setup phase will serve as a basis on which the CIAM will build its future outcomes.

In cases, where the home user has to configure the initial setup phase, he/she can assign priorities to specific UEs, which frequently utilize the femtocell for acquiring access to mobile services [e.g., the home user can define his/her CSG and determine the context in which a nearby macro UE can connect to the femtocell without degrading the quality of experience (QoE) of CSG's users]. Another priority assignment procedure can take place for different service classes (e.g., the home user can define which type of services will have the highest priority and thus no QoS degradation is acceptable even in high backhaul capacity utilization situations). Given the fact that a mentality shift occurs in cellular operators' industry towards giving several motivations to femtocell owners in order their FAPs to serve more macro UEs and thus offload mobile traffic from cellular operators' infrastructure, the initial setup phase can become very important for the femtocell owner to experience considerable bill discounts.

In cases, where ICT specialists configure the initial setup process (e.g., public access and enterprise scenarios analyzed in [11]), the whole procedure can become even more complicated as hybrid or open access femtocells are assumed and thus service classes' and user groups' priorities should be configured taken into consideration the overall HetSNets environment.

\subsection{Monitoring and gathering}

During operation, the CIAM, through the monitoring and gathering processes, will keep track of the statistical properties of the incoming traffic load as well as of the backhaul capacity in order to be able to adjust, if needed, the values initially selected by the user. For example, capacity adjustments and the various partitions' restructure can happen in cases of overload-state situations or in cases when context-aware prioritization based on the type of services that the femto-relay is currently supporting, occur. More specifically, if wired backhaul experiences intolerable delays (e.g., in overload-state situations or when delay-sensitive services cannot effectively be delivered), then the wireless backhaul is selected, as the required QoS levels can be more easily maintained, because of the fact that the data "remains" within one cellular operator's network.

The first sub-functionality of monitoring is the sensing of context, meaning both the detection and reception of data from sensors without introducing additional traffic overhead. Efficient scanning is a matter of vital importance since real-time limitations have to be satisfied and QoE objectives should be maintained within acceptable levels [9]. The key objective is the dynamic adjustment of the defined set of parameters by adopting event-based and on-demand monitoring policies [26]. One example of monitoring is the sensing of the FAP's radio environment in order to clarify if interference levels are below some predefined and desirable thresholds. If this is the case, then the relay module can inform CIAM about the wireless backhaul link alternative and consequently the CIAM will update the wired backhaul capacity partitions (i.e., PPA process described in Section 4), so that the latter can be restructured in favor of the QoS/QoE metrics for UEs. Femto-relay concept can also help in interference protection both in downlink and uplink scenarios. In downlink, a macro UE that has a weak direct link with eNB and is located in the vicinity of an open-access femto-relay can have an increased SINR if it selects to cooperate. Hence, the number of situations where twotier interference could lead in outages can be reduced. In uplink, a macro UE that transmits with full power to maintain its connection with the eNB can instead choose a two-hop transmission through an open-access femtorelay. This cooperation will result in power reduction by the macro UE and incurred interference in nearby closed-access or non-selected femto-relays will be mitigated.

Another monitoring/gathering example is when CIAM monitors CAM and ISAC modules. More specifically, when a significant traffic load variation occurs or if the backhaul capacity utilization is relatively low or specific delay constraints for real-time services have to be met, CIAM decides the extent of wired/wireless backhaul usage based on the context information that receives from the relay module, too. Although the RRM process can dynamically be adapted to small variations of the traffic load distribution through the PPA process, the outcome of CIAM is essential for the efficiency of the RRM operation under traffic load variations taking place in larger timeframes. Equally important is the outcome of CIAM for the activation or deactivation of the relay module (cf. Section 7). More specifically, when a femtocell's utilization is relatively low at specific time intervals (cf. available femtocell case depicted in Figure 5), CIAM triggers the relay module's operation (cf. Figure 2) and thus the femtocell can act as a relay allowing neighboring macro UEs to transmit in a two-hop fashion.

\section{Decode-and-forward relay module}

As we focus our interest on the uplink case, we identify the macro UEs as the main source of interference towards the femto BSs. This scenario is depicted in Figure 5, where two macro UEs are in the vicinity of four femtocells. Aiming to improve the SINR at the femto BSs, we propose to take advantage of femto BSs that currently do not serve any femto UE and to utilize them as relays for the macro UE. In this way, we can achieve reduction in transmission power of the macro 


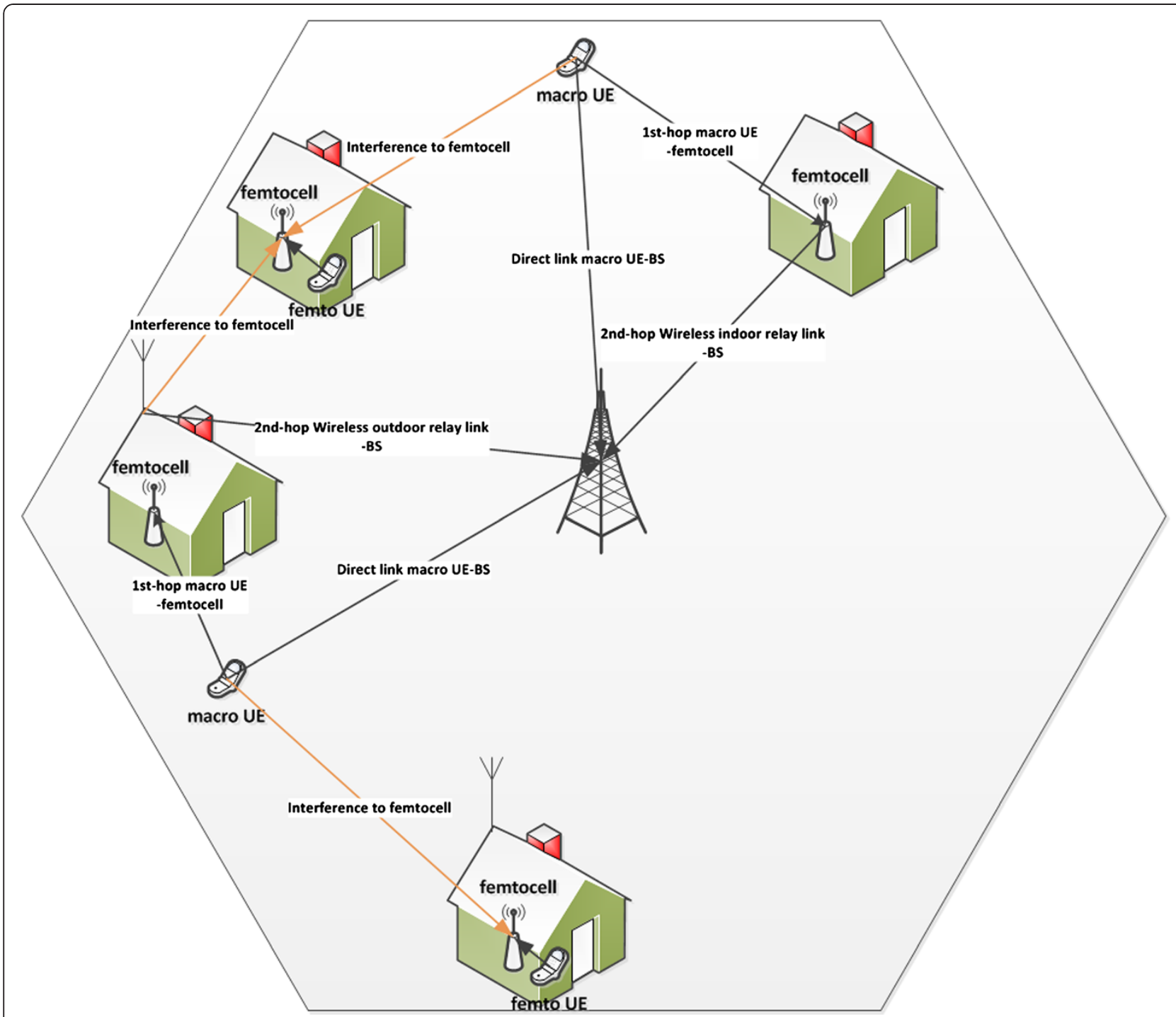

Figure 5 Interference in the uplink.

UE compared to the power required for direct transmission. By decreasing the transmission power, the neighboring femtocells, which were not available to cooperate, will experience less macro interference, too.

Moreover, in conjunction with the backhaul protection schemes analyzed in Section 4, we suggest employing outdoor donor antennas towards the macro BS, enhancing the femto BS with wireless relaying capabilities. This extra wireless backhaul does not replace the existing wireline backhaul but rather complements it. In this way, new possibilities for better QoS provision to delay sensitive applications as well as better interference coordination between two-tier networks are opened. The selection of outdoor antennas in the backhaul link increases the probability of having LOS conditions with the macro BS, resulting in reduced transmission power and less interference to nearby femtocells. In the case where no outdoor antenna is installed the indoor antenna could be used but with increased pathloss towards the macro BS. Furthermore, by choosing to route the macro UE traffic through the wireless backhaul, further protection and better usage of the wireline backhaul capacity of the femtocells is achieved, because more capacity (i.e., bandwidth) is available for UEs served by the wired backhaul. More specifically, indoor users connected with a different interface to the femto BS will not have to share their backhaul capacity with UEs, which do not belong to the CSG [27].

In this case, the UE that is located in the femtocell's vicinity will receive a message denoting the latter's availability to cooperate. As the femtocell's location is fixed, it can acquire the channel state information (CSI) of the wireless backhaul link towards the macro BS with good accuracy. This information is included in the message 
sent to the UE together with the CSI of the femto BSUE link. By receiving this information the UE has to answer the following question: For the data rate required by its service, which route will provide the largest power reduction? In order for the UE to decide its uplink route, a comparison between the direct and the two-hop links is performed.

From the above description, we see that the exploitation of the spatial diversity provided by the deployment of femtocells could lead to a two-hop transmission requiring reduced transmission power compared to a direct transmission towards the macro BS. As a result, having an additional path to choose from may effectively reduce the interference arising from the transmissions of the macro UE.

In our system, we assume only pathloss and lognormal shadowing effects assuming the suggested parameters in [28]. The frequency used for the communication is $2 \mathrm{GHz}$, the height of the BS antenna is $32 \mathrm{~m}$, while that of the $\mathrm{RN}$ is $10 \mathrm{~m}$. For the indoor antenna we consider a height half to that of the RN at $5 \mathrm{~m}$ and finally, the UE antenna's height is at $1.5 \mathrm{~m}$. Also, each link exhibits a different shadowing standard deviation with each case shown in Table 1. Moreover, for the link between the UE and the indoor femto BS antenna, we consider that an additional penetration loss equal to $20 \mathrm{~dB}$ is added in the link budget calculations. Following the technical specifications of [28], the pathloss of the direct link between a macro UE and the macro BS is given by

Table 1 Simulation parameters

\begin{tabular}{ll}
\hline Parameter & Value \\
\hline Macrocell radius (m) & 500 \\
Femtocell radius (m) & 50 \\
Number of femtocells & $0-100$ \\
Number of macro UEs & 25 \\
Modulation & UE-Femto BS link: \\
& Adaptive modulation \\
Femto BS-Macro BS link: & (QPSK, 16 QAM) \\
Adaptive modulation & \\
(QPSK, 16 QAM) & \\
Shadowing std UE-Macro BS (dB) & 10 \\
Shadowing std UE-Femto BS (dB) & 10 \\
Shadowing std femto BS-macro BS (dB) & 6 \\
UE transmit SNR (dBm) & 23 \\
Femto transmit SNR (dBm) & 23 \\
Receiver diversity gain (dB) & 3 \\
Penetration loss (dB) & 20 \\
Results & Power reduction, \\
\hline
\end{tabular}

$$
P L(R)_{\mathrm{UE}-\mathrm{BS}}=131.1+42.8 \log _{10}(R)
$$

where $R$ is the distance in kilometers between the transmitter and the receiver. The shadowing standard deviation is equal to $10 \mathrm{~dB}$. In (11), we take under consideration the non-line-of-sight (NLOS) case, which in an urban setting of UEs, maintains, most of the time, NLOS connectivity with the macro BS.

For the two-hop transmission, in the link between the macro UE and the cooperating femto BS, NLOS conditions will be dominant, as the access antennas of the femtocells will be located inside buildings. As a result, the pathloss will be [28]

$$
P L(R)_{\mathrm{UE}-\mathrm{fBS}}=128.1+37.6 \log _{10}(R)
$$

The shadowing standard deviation has a value of 10 $\mathrm{dB}$. In the second hop, when an outdoor antenna is installed, the pathloss of the femto BS-macro BS link is calculated based on [28]

$$
P L(R)_{\mathrm{fBS}-\mathrm{BS}}=100.7+23.5 \log _{10}(R)
$$

In this case, we assume line-of-sight (LOS) conditions as the outdoor antenna will be on the rooftop and directed towards the macro BS. The shadowing standard deviation is equal to $6 \mathrm{~dB}$.

On the contrary, when no outdoor antenna is used, the link is assumed to be NLOS and the pathloss is given by [28]

$$
P L(R)_{\mathrm{fBS}-\mathrm{BS}}=131.1+42.8 \log _{10}(R)
$$

In this link, the shadowing standard deviation has a greater value, equal to $10 \mathrm{~dB}$.

\section{Simulation results}

In this section, we aim to evaluate, through event driven simulation, the two main functionalities that are introduced by the proposed modules which are (a) the ability of the RRM integration layer to provide a predetermined QoS even under varying traffic loads and (b) the ability of the relay module to contribute to the reduction of the overall local interference. For (a), we evaluate the performance of the RRM integration layer through a custom made event-driven simulator written in $\mathrm{C} / \mathrm{C}++$. As already referred, we assume that the incoming service requests arrive according to a Poisson process, while their duration is exponentially distributed. For (b), we followed the simulation guidelines in [28] for the development of an event-driven simulator implemented in Matlab assuming the simulation parameters shown in Table 1. 


\subsection{Perceived QoS under varying traffic loads}

In this section, we study the ability of the CA-FEI scheme to continuously adjust to variations of the traffic load distribution. Hybrid Partitioning Schemes (HPS) and Fixed Partitioning Schemes (FPS) [13] can be considered as a base of reference for comparison because their operation can be approximated, up to some point, with the currently available in the market router and femtocell combinations. However, HPS offer better capacity utilization and are more immune against traffic variations compared to FPS and are thus better competitors for the proposed CA-FEI scheme. In our case, the employed HPS has a fixed partition, defined for each service, which requires specific QoS guarantees, while the remaining capacity is utilized as common pool partition for all service calls.

We assume a simulation scenario, where a femtocell coexists with a wire-line IP network sharing a 100-Mbps fast ethernet backhaul line. The period $\Delta t$ of PPA is set to $100 \mathrm{~ms}$, the guarding factor is uniformly set to 10 and the averaging time interval $d$ is extended up to the duration of the simulation (i.e., the blocking rate $B$ is recalculated each time PPA is executed). Both $C A-F E I$ and HPS schemes arrange their capacity partitions expecting at the worst case a $35 \%$ reduction of the backhaul capacity as well as a traffic load distribution consisting of only delay sensitive services (40\% Voice, 30\% Video-telephony, and 30\% Streaming video) all having a target GoS of $1 \%$. In order to analyze the effect of PPA function, we further assume that the traffic load distribution presents an unpredictable short-term variation. Thus, the percentage of video-telephony calls is increased to $50 \%$, while the percentage of streaming video calls is decreased to $10 \%$. The following simulation results correspond to a confidence interval of less than $5 \%$ with a confidence level of $95 \%$.

Due to the assumed worst case scenario and the strict required GoS, the capacity left for the common pool partition is significantly reduced for both HPS and CA-FEI schemes. Thus, the HPS scheme cannot cope with the unexpected variation of the traffic load. As a result, the blocking probability of video-telephony calls ranges approximately between 52 and $58 \%$ when the backhaul capacity is highly utilized (as shown in Figure 6). At the same time, the reduced streaming video traffic makes use of a capacity partition that is initially designed to handle a significantly higher traffic volume. Consequently, streaming video calls have a zero blocking probability.

On the other hand, when the CA-FEI scheme is employed, video-telephony calls are able to utilize the capacity, which remains unused at the partition of streaming video calls. Consequently, the blocking probability of video-telephony is significantly reduced compared to HPS and remains below the required GoS of
$1 \%$ for traffic loads up to $94 \%$. At the same time, due to the employed guarding factor, the blocking probability of the streaming video calls remains also below $1 \%$ at all traffic loads, even though their partition is in this case fully utilized. The efficiency of the CA-FEI scheme can also be verified at Table 2, where it is shown that CAFEI offers increased capacity utilization compared to the HPS scheme.

In conclusion, at high traffic loads, HPS (and consequently FPS, used in current femtocell deployments) are inefficient in terms of capacity utilization. Hence, considering the increased traffic volume expected for LTE systems, we have proposed a framework, which provides a suitable environment for the femtocell operation through effective and efficient management of the total incoming traffic and the common limited backhaul capacity.

\subsection{Impact of DF relay module on the overall system's performance}

In order to study the efficiency of the proposed scheme in the uplink of a cellular network, we developed a simulator in Matlab considering [28] for the simulation parameters. The simulation parameters are listed in Table 1. We consider a macro cell with radius $500 \mathrm{~m}$ operating in an urban environment with the macro BS located in the center and a varying number of femtocells that are available for cooperation. The macro BS is equipped with two receive antennas providing a diversity gain of $3 \mathrm{~dB}$. The femtocells have a coverage radius of $50 \mathrm{~m}$ and are uniformly located in the macrocell. The reference receiver sensitivity values are calculated using the values in Table 22.6 of [29], considering a bandwidth of $20 \mathrm{MHz}$ and the following equation [29]

$$
\begin{aligned}
\text { REFSENS }= & k T B+\mathrm{NF}+\mathrm{SINR}+\mathrm{IM} \\
& -3(\mathrm{dBm})
\end{aligned}
$$

where $k T B$ is the thermal noise level equal to -174

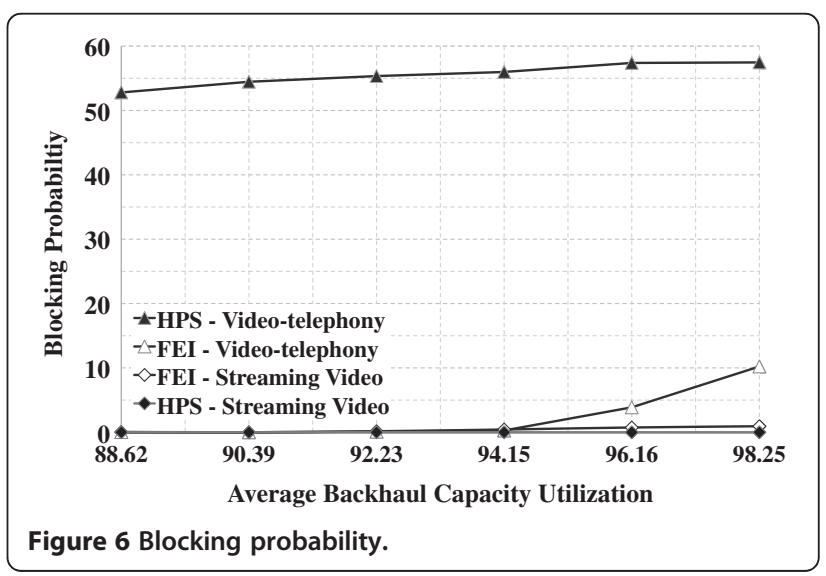


Table 2 Total capacity utilization

\begin{tabular}{|c|c|c|c|c|c|c|}
\hline Offered traffic load & 88.62 & 90.39 & 92.23 & 94.15 & 96.16 & 98.25 \\
\hline FEl & 80.16 & 81.12 & 82.27 & 83.24 & 84.19 & 87.29 \\
\hline HPS & 47.7 & 48 & 48.61 & 48.88 & 49.07 & 50.38 \\
\hline Relative percentage of increase & 68.05 & 68.99 & 69.24 & 70.3 & 71.57 & 73.24 \\
\hline
\end{tabular}

$\mathrm{dBm} / \mathrm{Hz}, \mathrm{NF}$ is the prescribed maximum noise figure for the receiver and IM is the implementation margin while $-3 \mathrm{~dB}$ is the diversity gain. The modulation schemes range from QPSK $1 / 3$ to 16QAM 4/5 and we use the corresponding SINR and IM values from Table 22.6 of [29].

As mentioned in Section 7, the femtocells are assumed to have an outdoor donor antenna to support the wireless backhaul link towards the macro BS and a classic indoor coverage antenna. For simplicity, we assume NLOS conditions for UE-to-macro BS and UE-to-femto BS and LOS conditions for the femto BS-to-macro BS links. The UEs are also dropped uniformly in the macrocell area and are equipped with one transmit antenna. As a result, the maximum modulation order they can support is 16 QAM. Whenever a two-hop transmission is selected, a penetration loss of $20 \mathrm{~dB}$ is considered [28]. Also, overhead due to signaling when a macro UE is hand-offed from the macro BS to a femto BS and vice versa is considered negligible in the data rate results that are presented in the following paragraphs.

To evaluate the performance of the proposed scheme, we simulate the above topology to obtain numerical results regarding: (a) power reduction in the transmission of the macro UEs and the backhaul antennas and (b) data rate achieved by them. For the first set of numerical results, we set a target data rate of 14.4 Mbps for the uplink as it is a reasonable value considering QoS fairness even for cell edge users. As a reference scheme is used the classic femtocell setup, where only users of the CSG are served and there is no capability to cooperate with other UEs. Results of the simulation are depicted in Figure 7 for the increasing percentage of available femtocells. Note that the values for power reduction are the differential gain of the proposed solution compare to the classic femtocell architecture.

We observe that as the available femtocells increase, there is a greater chance to achieve a transmission with reduced power by the interfering macro UEs and the backhaul antennas positioned on the roof of the building. The decrease in $\mathrm{dBm}$ ranges from 2.9 to $9.07 \mathrm{dBm}$ for the extreme case, where all the femtocells are available (cf. Figure 5). For medium cooperation factors (i.e., 30-50\%), we see that power reduction takes values between 4.5 and $6 \mathrm{dBm}$. As a result, to achieve the specified data rate, macro interference can be reduced whenever an additional path is available to select from (i.e., when a cooperating femtocell is near a macro UE). Moreover, the arising interference to femtocells currently serving UEs of CSG will be accordingly reduced.

In the next simulation, we assume the cases of 30 and $40 \%$ availability of cooperating femtocells and we examine the possibility of performing the second hop towards the macro BS through the wireline backhaul in case it can be allocated for macro UEs traffic. Figure 8 shows the results for varying percentage of wired transmissions by the cooperating femtocells for the link between the femto BS and the macro BS.

From Figure 8, we observe that for each case, if the wired backhaul is available, we completely avoid the interference in the second hop. As a result, we see an improvement over the case of only wireless backhaul, which for the case of $30 \%$ has a range between 4.4 and $6.15 \mathrm{dBm}$ and for the case of $40 \%$ between 5.1 and 7.3 $\mathrm{dBm}$.

In Figure 9, we present the numerical results for the data rate improvement of macro UEs in order to examine whether CA-FEI scheme can provide gains in this area, compared to the classic femtocell setup. In order to explore the increase in the data rate of the macro UEs, the latter adopt a policy of selecting the best endto-end path and transmitting with the best possible data rate. This comes in contrast to the power reduction policy of the previous simulation. To measure the end-to -end capacity, we employ the half-duplex constraint in the two-hop transmission and the capacity is given by [30]

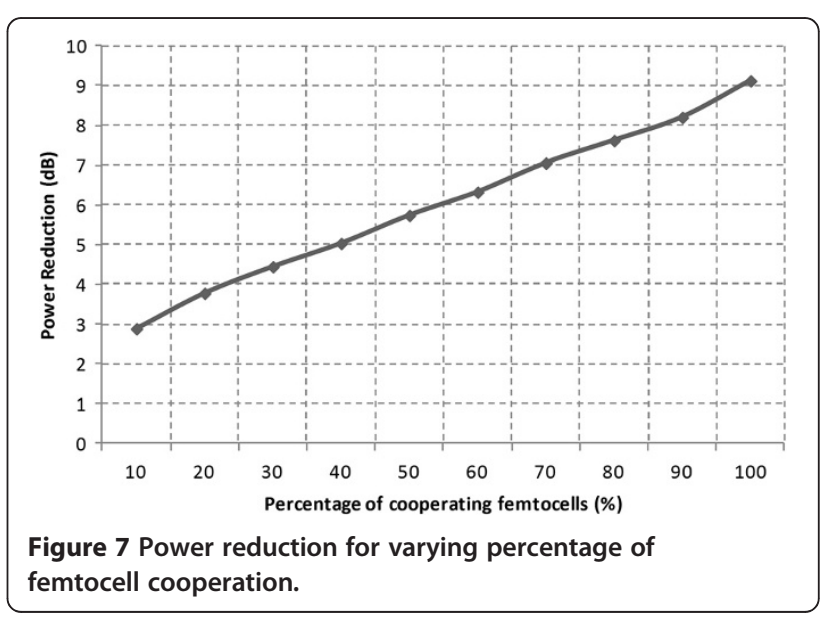




$$
C_{\text {two-hop }}=\frac{1}{2} \min \left(C_{\text {first-hop }}, C_{\text {second-hop }}\right)
$$

We see that when a macro UE receives an availability message from a femtocell with the best link, it can associate with it and relay its traffic through a two-hop link. As we mentioned before, the two-hop route will be selected, when the direct link's data rate is below the two-hop's one. Hence, by exploiting spatial diversity, the macro UEs can improve their data rate by $26 \%$ in the extreme case of $100 \%$ available femtocells. For more realistic cases of $30-50 \%$ femtocell availability, the improvement can range between 10 and $16 \%$. We note that these values are the relative improvement achieved with our scheme compared to the classic femtocell setup.

Finally, we study the case where an outdoor antenna for the wireless backhaul is not always available. As one of the main benefits of femtocell deployment is their plug and play nature, studying the effectiveness of femtocell cooperation in cases where outdoor antennas are available in realistic percentages will give us an indication of the usefulness of the CA-FEI scheme. More specifically, Figure 10 presents the data rate performance of macro UEs in the cases of 30 and $40 \%$ availability of cooperating femtocells for a varying percentage of installed outdoor antennas.

We see that macro UEs can benefit from femtocell cooperation in cases where the wireless backhaul is supported by outdoor antennas. When no outdoor antennas are available, data rate sees almost no improvement due to the low transmit power of the femtocell and the penetration loss. On the other hand, when outdoor antennas are added to the network, we see significant improvement even for percentages below 50\%. In the low percentage range, we observe that data rate increases between 1.3 and $5.5 \%$ for $30 \%$ femtocell availability,

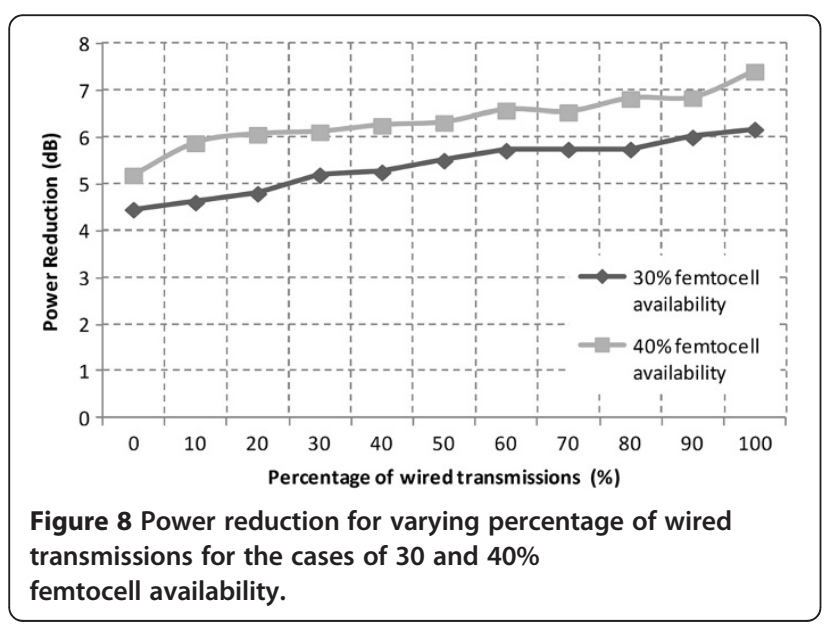

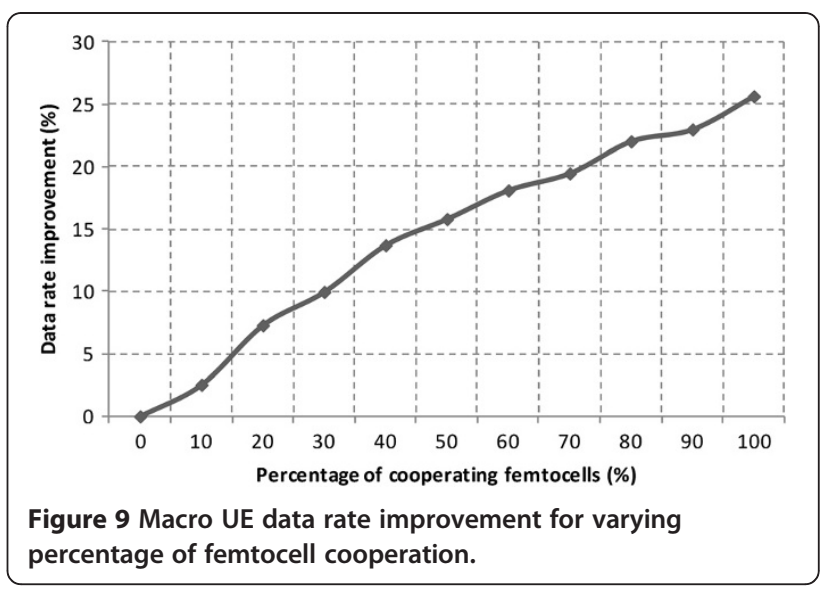

while for $40 \%$ femtocell availability, we see an increase of 1.8 to $6.7 \%$ compared to the case of only direct transmission in the uplink. Again, the above percentages are relative improvement compared to the reference case of the classic femtocells without wireless backhaul.

\section{Conclusions and future work}

This article elaborates on aspects of the recently introduced communication paradigm of HetSNets, which currently constituted the study item of 3GPP LTE-Advanced releases. Among a plethora of research issues examined in the HetSNets area, we dealt with open challenges such as the femtocells' limited backhaul capabilities and the macrocell/femtocell cooperation by proposing networking resources and interference management solutions from the femtocell point of view. Therefore, a novel contextaware framework for the efficient integration of femtocells in IP and cellular infrastructures, called CA-FEI, is proposed. CA-FEI exploits context-aware mobile and wireless networking principles thus providing a controlled architectural environment, in which a femtocell is able to function properly. Simulation results show that CA-FEI outperforms competitive schemes used in real-market

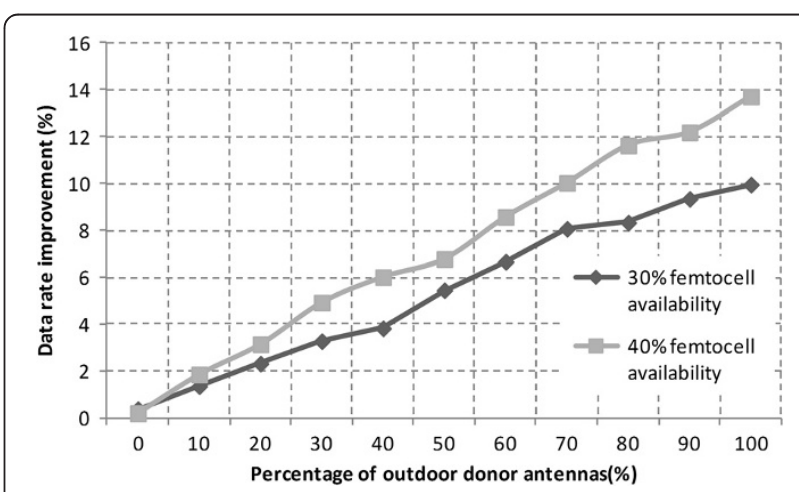

Figure 10 Macro UE data rate improvement for varying percentage of femtocell cooperation. 
femtocell deployments regarding QoS, energy savings, and data rate enhancements achieving to (a) provide a predetermined QoS even under varying traffic loads via the RRM integration layer and (b) contribute to the reduction of the macrocell/femtocell interference via the relay module.

Regarding business-logic threats, cost efficiency and the real-market penetration rate are the major challenges for the proposed femto-relay concept. Hence, the femto-relays' manufacturing and large-scale deployment should outperform traditional deployment solutions (e.g., operator-initiated fully-fledged eNBs' and userinitiated uncoordinated femtocells installation) in terms of OPEX/CAPEX and delivered QoS/QoE, while new business-logic research pathways and noteworthy expected impact should be realized for all femto-relay beneficiaries. More specifically, cellular operators can exploit femto-relaying towards managing whole small cell networks being under their realm as a single HetSNets entity and thus be able to simultaneously tackle challenges on heterogeneous access and backhaul parts of their infrastructure. Mobile service providers can also increase their revenues by exploiting promising femto-relaying capacity gains, towards delivering novel and even more resource-consuming mobile applications. Vendors/manufacturers can benefit from developing elegant $H / W$ solutions and customer-friendly small cell network products. Research and retail-oriented SMEs can also benefit from the envisioned femto-relay market growth, while citizens will be provided with a wider selection of mobile applications with better QoS, seamless service provisioning, and service outages minimization.

Based on the above considerations and regarding our related future work, we plan to extend the CA-FEI concepts applicability to larger-scale HetSNets environments investigating its flexibility to various HetSNets deployment scenarios. We aim to prove that operating femtocells as relays can improve HetSNets' overall RRM objectives by extending the overall virtual backhaul link capacity in small cell areas, where excessive traffic is monitored at specific time intervals and that optimal exploitation of context information among all HetSNets entities can be the cornerstone of the proposed architectural solutions.

\section{Competing interests}

The authors declare that they have no competing interests.

\section{Acknowledgments}

This work has been partially funded by the Research Projects GREENET (PITN-GA-2010-264759), CO2GREEN (TEC2010-20823), GREEN-T (CP8-006).

\section{Author details}

'Department of Information and Communication Systems Engineering, University of the Aegean, Karlovassi Samos GR-83200, Greece. ${ }^{2}$ Department of Electronic and Electrical Engineering, University of Sheffield, Western Bank,
Sheffield S10 2TN, UK. ${ }^{3}$ Centre Tecnologic de Telecomunicacions de Catalunya (CTTC), Castelldefels, Barcelona, Spain.

Received: 29 June 2012 Accepted: 31 January 2013

Published: 5 March 2013

\section{References}

1. D Lopez-Perez, I Guvenc, G De La Roche, M Kountouris, TQS Quek, J Zhang, Enhanced intercell interference coordination challenges in heterogeneous networks. IEEE Wirel. Commun. Mag. 18(3), 22-30 (2011)

2. D Martin-Sacristan, JF Monserrat, J Cabrejas Penuelas, D Calabuig, S Garrigas, N Cardona, On the way towards fourth-generation mobile: 3GPP LTE and LTEAdvanced. EURASIP J. Wirel. Commun. (2009), 1-10 (2009). doi:10.1155/2009/354089

3. J Zhang, G De La Roche, Femtocells: Technologies and Deployment (Wiley, New York, 2010). ISBN 978-0470742983

4. V Chandrasekhar, J Andrews, A Gatherer, Femtocell networks: a survey. IEEE Commun. Mag. 46(9), 59-67 (2008)

5. O Tipmongkolsilp, S Zaghloul, A Jukan, The evolution of cellular backhaul technologies: current issues and future trends. IEEE Commun. Surv. Tutor. 13(1), 97-113 (2011)

6. P Kulkarni, WH Chin, T Farnham, Radio resource management considerations for LTE femto cells. ACM SIGCOMM Comput. Commun. Rev. 40(1), 26-30 (2010)

7. G De, A la Roche, DL-P Valcarce, J Zhang, Access control mechanisms for femtocells. IEEE Commun. Mag. 48(1), 33-39 (2010)

8. P Xia, V Chandrasekhar, JG Andrews, Open vs. closed access femtocells in the uplink. IEEE Trans. Wirel. Commun. 9(12), 3798-3809 (2010)

9. P Makris, DN Skoutas, C Skianis, A survey on context-aware mobile and wireless networking: on networking and computing environments integration. IEEE Commun. Surv. Tutor. 15(1), 362-386 (2013)

10. 3GPP, LTE, Service Requirements for Home NodeBs (UMTS) and eNodeBs (LTE) 3GPP TS 22.220, V.10.3.0, Release 10, 2010

11. DN Skoutas, P Makris, C Skianis, Optimized Admission Control Scheme for Coexisting Femtocell, Wireless and Wireline Networks (Springer Telecommunication Systems, Special Issue on Mobility Management in Future Internet, 2012). in press

12. MZ Chowdhurry, S Choi, YM Jang, KS Park, Dynamic SLA negotiation using bandwidth broker for femtocell networks, in Proceedings of 1st International Conference on Ubiquitous and Future Networks (ICUFN), 2009, pp. $12-15$

13. A Rouskas, DN Skoutas, G Kormentzas, DD Vergados, Code reservation schemes at the forward link in WCDMA. Elsevier Comput. Commun. 27(9), 792-800 (2004)

14. R Skehill, M Barry, W Kent, M O'Callaghan, N Gawley, S Mcgrath, The common RRM approach to admission control for converged heterogeneous wireless networks. IEEE Wirel. Commun. 14(2), 48-56 (2007)

15. E Stevens-Navarro, AH Mohsenian-Rad, $\vee$ Wong, Connection admission control for multiservice integrated cellular/WLAN system. IEEE Trans. Veh. Technol. 57(6), 3789-3800 (2008)

16. D Calabruig, JF Monserrat, DM Sacristan, N Cardona, Joint dynamic resource allocation for QoS provisioning in multi-access and multi-service wireless systems. Springer MONET 15(5), 627-638 (2010)

17. N Andrews, Y Kondareddy, P Agrawal, Channel Management in Collocated WiFi-WiMAX Networks, in Proceedings of 42nd Southeastern Symposium on System Theory (SSST), 2010, pp. 133-137

18. T Elkourdi, O Simeone, Femtocell as a relay: an outage analysis. IEEE Trans. Wirel. Commun. 10(12), 4204-4213 (2011)

19. A Rath, S Hua, SS Panwar, FemtoHaul: Using femtocells with relays to increase macrocell backhaul bandwidth, in Proceedings of INFOCOM IEEE Conference on Computer Communications Workshops, 2010, pp. 1-5

20. Z Dizhi, S Wei, Interference-controlled load sharing with femtocell relay for macrocells in cellular networks, in Proceedings of Global Telecommunications Conference (GLOBECOM), 2011, pp. 1-5

21. A Tyrrell, F Zdarsky, E Mino, M Lopez, Use Cases, Enablers and requirements for evolved femtocells, in Proceedings of Vehicular Technology Conference (VTC Spring), 2011, pp. 1-5

22. A Adhikary, V Ntranos, G Caire, Cognitive femtocells: breaking the spatial reuse barrier of cellular systems, in Proceedings of Information Theory and Applications Workshop (ITA), 2011, pp. 1-10 
23. 3GPP, Technical Specifications Group Services and Systems Aspects; End-to-end Quality of Service (QoS) Concept and Architecture. 3GPP TS 23.107, V9.1.0, Release 9, 2010

24. S Blake, D Black, M Carlson, E Davies, Z Wang, W Weiss, An architecture for differentiated services. Internet RFC 2475, 1998

25. B Statovci-Halimi, G Franzl, QoS Differentiation and Internet Neutrality-A Controversial Issue within the Future Internet Challenge (Springer Telecommunication Systems, 2011)

26. S Kang, J Lee, H Jang, Y Lee, S Park, J Song, A scalable and energy-efficient context monitoring framework for mobile personal sensor networks. IEEE Trans. Mob. Comput. 9(5), 686-702 (2010)

27. IF Akyildiz, DM Gutierrez-Estevez, E Chavarria Reyes, Femto-relay systems and methods of managing same. US Patent 20120076027, 2012, http://users.ece. gatech.edu/ dmge3/publications/patent1.pdf

28. 3GPP, Technical Specifications Group Radio Access Network, Evolved Universal Terrestrial Radio Access (E-UTRA); Further advancements for E-UTRA physical layer aspects. 3GPP TR 36.814, V9.0.0, Release 9, 2010

29. S Sesia, I Toufik, M Baker, LTE The UMTS Long Term Evolution, From Theory to Practice (Wiley, New York, 2009). ISBN 978-0-470-69716-0

30. JN Laneman, DNC Tse, GW Wornell, Cooperative diversity in wireless networks: efficient protocols and outage behavior. IEEE Trans. Inf. Theory 50(12), 3062-3080 (2004)

doi:10.1186/1687-1499-2013-62

Cite this article as: Makris et al: A context-aware framework for the efficient integration of femtocells in IP and cellular infrastructures. EURASIP Journal on Wireless Communications and Networking 2013 2013:62.

\section{Submit your manuscript to a SpringerOpen ${ }^{\circ}$ journal and benefit from:}

- Convenient online submission

- Rigorous peer review

- Immediate publication on acceptance

- Open access: articles freely available online

- High visibility within the field

- Retaining the copyright to your article 